\title{
Traitement \\ de l'information \\ en matière \\ de recherche \\ dans le domaine \\ de la santé
}

Nul n'est censé

ignorer la loi !

Catherine Bonaïti-Pellié, Patrick Arveux,

Agathe Billette de Villemeur, Nicolas Dantchev,

Alexis Elbaz, Elisabeth Fabre-Guillevin,

Jeanne Fresson, Christine Saura,

Marie-Josèphe Saurel-Cubizolles,

Jean-Louis Serre, Dominique Simon,

Isabelle Stucker, Bertrand Thélot,

Pascale Tubert-Bitter, Mahmoud Zureik

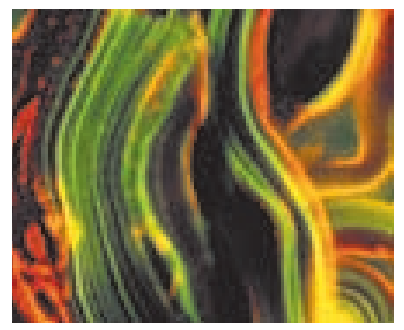

C. Bonaïti-Pellié : présidente du CCTIRS, Inserm U535, BP 1000, 94817 Villejuif Cedex, France. catherine.bonaiti@inserm.fr
Le $1^{\text {er }}$ juillet 1994, la loi 94-548 ajoutait un chapitre Vbis (maintenant IX) à la loi du 6 janvier 1978 relative à l'informatique, aux fichiers et aux libertés (modifiée par la loi relative à la protection des personnes physiques à l'égard des traitements de données à caractère personnel du 6 août 2004), et instituait pour son application le Comité consultatif pour le traitement de l'information en matière de recherche dans le domaine de la santé (CCTIRS), dont la composition et la mission sont énoncées dans l'article 54 du chapitre IX [1-3].

Le rôle du CCTIRS est d'éclairer la CNIL (Commission nationale de l'informatique et des libertés) sur la justification du traitement de données à caractère personnel dans un but de recherche, et, pour cela, d'émettre un avis sur la méthodologie de la recherche. En fonction de cet avis, la CNIL pourra ou non se prononcer sur la nécessité de déroger au secret professionnel qui protège les données de santé à caractère personnel et autoriser le traitement de données proposé.

Les co-auteurs de cet article sont tous membres du CCTIRS.
Au fil du temps et des lois, les missions du CCTIRS ont été modifiées, sa manière de fonctionner a évolué, mais le principe est toujours celui de protéger l'individu, non dans son intégrité physique, ce dont sont chargés les comités de protection des personnes (CPP) [4, 11], mais de la divulgation des données concernant sa santé, qui sont considérées comme des données sensibles au même titre que les données concernant les origines raciales ou ethniques, les opinions philosophiques, politiques, syndicales, religieuses, ou la vie sexuelle des personnes, ainsi que mentionné dans l'article 8 de la loi [1].

À la différence des CPP, le CCTIRS est national: les 15 membres qui le composent sont donc en charge de l'examen de toutes les recherches utilisant des données à caractère personnel dans le domaine de la santé en France.

Les membres du comité constatent régulièrement, lors de leur pratique quotidienne, que les traitements qui devraient leur être soumis... ne le sont pas toujours. La plupart du temps, cette lacune est due à une mécon- 
naissance de nombreux médecins et chercheurs de la législation en matière de traitement des données à caractère personnel. S'il est admis que nul n'est censé ignorer la loi, force est de constater que peu de gens la connaissent vraiment, d'autant que ces dernières années ont été riches en évolutions multiples, ce qui n'en facilite pas toujours la lecture. II est tout de même inquiétant de constater, après les efforts didactiques développés par la CNIL lors de la révision de la loi Informatique et Libertés en 1994 et de sa mise à jour en 2004 [2, 3], que de nombreuses personnes continuent de confondre données anonymes et anonymisées, et estiment qu'un fichier ne relève pas de la loi si les personnes sont identifiées par un simple numéro d'ordre.

Trente ans après la loi relative à l'informatique, aux fichiers et aux libertés [1] et 20 ans après la loi sur la protection des personnes qui se prêtent à des recherches biomédicales [4], l'objectif de cet article est de mieux faire connaître les règles sur la protection des données de santé, de sensibiliser les professionnels de santé et les chercheurs sur l'intérêt de la mission du comité qui participe à la protection des personnes et de lui permettre de rendre compte de son activité dans un souci de transparence vis-à-vis des équipes de recherche.

\section{Composition et champ d'intervention du CCTIRS}

Le CCTIRS est composé de 15 membres, dont un président, qui sont des médecins et des scientifiques compétents dans les domaines de l'épidémiologie, de la génétique et de la biostatistique. Ils sont nommés par arrêté conjoint du ministre chargé de la recherche et du ministre chargé de la santé. Ils se réunissent toutes les 4 semaines (avec une interruption pendant la période d'été) afin de pouvoir effectivement rendre leurs avis au bout du délai réglementaire d'un mois fixé par la loi.

Le comité était initialement envisagé comme un Haut Comité sur l'Information en Santé, capable de rendre des décisions au nom de l'intérêt public. Au fur et à mesure de l'élaboration du projet de loi, nombre d'objections ont été apportées à ces propositions. En effet, celui-ci ne devait déborder ni sur la CNIL qui conservait l'autorité, ni

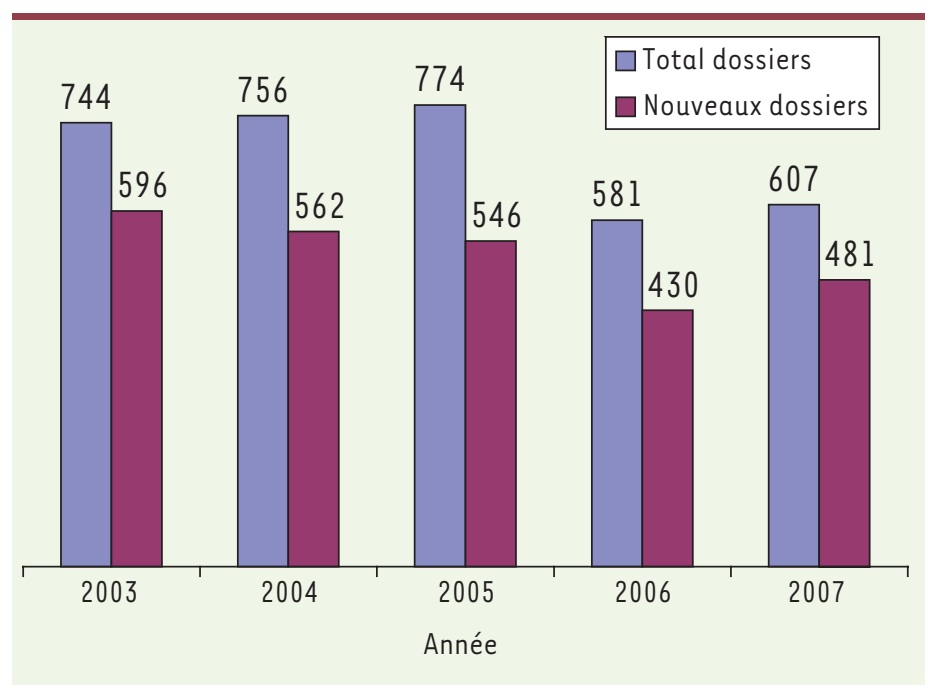

Figure 1. Nombres de dossiers examinés par le comité entre 2003 et 2007. sur les comités qui jugeaient de la protection des personnes, ni sur les comités scientifiques des promoteurs qui se portent garants de la valeur scientifique. Au final, il a été décidé que le comité serait une structure légère et réactive visant à enrichir la réflexion de la CNIL, et à l'aider dans sa décision d'autoriser ou non le traitement, en proposant un avis d'experts qui porte à la fois sur la nature des informations médicales utilisées et sur leur intérêt pour la recherche envisagée. C'est ainsi qu'en définitive la mission du CCTIRS n'inclut pas un avis sur la pertinence de la recherche, comme cela avait été initialement prévu. II est à noter que le comité est malgré tout régulièrement confronté à cette problématique et ce n'est qu'à regret que les scientifiques qui le composent s'interdisent de se prononcer sur l'intérêt d'une recherche. Étant donné la composition du comité et le nombre très restreint de ses membres, qui ne peuvent évidemment pas couvrir l'ensemble des domaines de recherche en santé, les membres sont conscients qu'il ne serait ni possible ni légitime de juger de la pertinence de tous les projets de recherches qui lui sont soumis.

\section{Procédure d'expertise, dossiers examinés et avis rendus}

L'ensemble des étapes de la procédure d'expertise est détaillé dans un document «Le CCTIRS mode d'emploi », rédigé en avril 2007 par les membres du comité et relu par la responsable de la division des affaires publiques et sociales à la CNIL. Son objectif est de faciliter la tâche des responsables de projets et de faire gagner du temps aux demandeurs comme aux évaluateurs. II est régulièrement mis à jour en fonction des lois qui se mettent en place. Le texte est téléchargeable sur le site du Ministère de l'enseignement supérieur et de la recherche (www.enseignementsup-recherche.gouv. fr/cid20537/cctirs.html).

Après une très forte augmentation (+80\%) entre 1997 et 2002 [5], le nombre de dossiers examinés par le comité a encore progressé, mais avec une franche inflexion en 2006, manifestement due à l'élargissement de la procédure simplifiée, comme nous le verrons plus loin (Figure 1).

Au cours de l'année 2007, les membres du comité consultatif se sont réunis 13 fois et ont examiné 607 dossiers. La répartition des avis est indiquée dans la Figure 2. Les avis sont en majorité favorables, assortis souvent de recommandations. Lorsque les critiques sont nombreuses, le Comité réserve son avis et le dossier est mis en attente. Les avis défavorables concernent les projets ne satisfaisant pas aux exigences minimales d'une recher- 


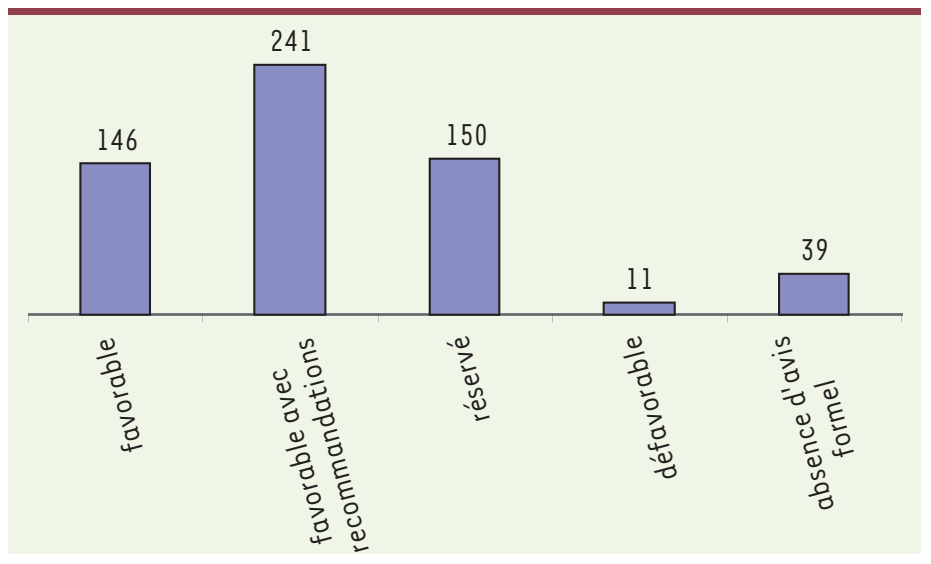

Figure 2. Avis du comité au cours de l'année 2007.

che dans le domaine de la santé. Les dossiers n'ayant pas reçu d'avis formel sont ceux pour lesquels le comité a considéré qu'ils ne relevaient pas de sa compétence (étude totalement anonyme, base de données sans projet de recherche, absence totale de projet de recherche dans le domaine de la santé...).

\section{La procédure simplifiée pour les recherches biomédicales}

Cette procédure homologuée par la CNIL a été mise en place en février 2006, pour simplifier le travail des porteurs de projets en leur évitant une soumission à de multiples comités. Il s'agissait d'une demande forte du CCTIRS [5] qui avait noté que ces soumissions multiples d'un même projet étaient problématiques, les comités concernés pouvant avoir éventuellement des avis divergents, et qui souhaitait que la procédure simplifiée, qui ne concernait jusqu'ici que certains essais cliniques, soit élargie à la majorité des recherches biomédicales, à condition que les CPP soient renforcés par des méthodologistes. La procédure simplifiée concerne les recherches biomédicales relevant des dispositions des articles L.1121-1 et suivants du code de la santé publique et qui doivent être obligatoirement soumises à un CPP [4]. Dans ce cas, les organismes adressent directement à la CNIL un engagement de conformité à la méthodologie de référence MR-001 pour les traitements de données personnelles opérés dans le cadre de recherches biomédicales (www.cnil.fr/index.php ?id=2365). Un certain nombre de traitements en sont exclus, en particulier ceux qui font apparaître l'identité complète de la personne, les recherches en génétique ayant pour objectif l'identification des personnes, et les recherches dont l'objectif principal est l'étude des comportements.

Depuis la mise en place de la procédure simplifiée, plusieurs études pouvant en bénéficier ont néanmoins été adressées au comité, la plupart du temps en raison d'une mauvaise connaissance de la procédure. Un problème particulier est celui des projets de recherche s'accompagnant de la constitution d'une banque d'ADN. Après discussion avec la CNIL, il s'avère que dès lors que la constitution de cette banque est directement justifiée par la recherche biomédicale envisagée, celle-ci bénéficie bien de la procédure simplifiée. Inversement, les exclusions à la méthodologie de référence ne sont pas toujours bien comprises des chercheurs ni même des organismes. C'est ainsi que certains membres du comité découvrent fortuitement, par exemple à l'occasion de propositions de collaboration, des recherches biomédicales qui auraient dû être soumises au comité car elles entraient dans l'un des motifs d'exclusion (étude d'une maladie psychiatrique, étude dans laquelle le nom complet des personnes apparaissait dans un fichier de recherche...), ne l'ont pas été : les responsables du traitement ont-ils considéré à tort que ces recherches étaient couvertes par l'engagement de conformité à la méthodologie de référence ou les responsables de la recherche ont-ils omis d'avertir leur organisme de leur recherche? C'est une question à laquelle le comité ne peut répondre pour l'instant avec les moyens dont il dispose. Nous engageons fortement les porteurs de projets de recherche et les promoteurs institutionnels à bien vérifier que la recherche qu'ils envisagent n'entre pas dans l'un des motifs d'exclusion à la méthodologie de référence.

\section{La protection des personnes dans les études observationnelles}

II y a quelques temps, F. Lemaire [6] attirait l'attention de la communauté médicale sur le problème rencontré par des auteurs français pour la publication de leur étude dans une revue américaine car ils n'avaient pas pu fournir l'avis favorable d'un comité d'éthique. $\varepsilon n$ effet, dans la mesure où ces études étaient purement observationnelles, elles ne requéraient pas l'avis d'un CPP. F. Lemaire soulignait alors l'inconvénient de ces apparentes disparités entre pays, les revues américaines exigeant un avis éthique dans des études qui, selon la loi française, n'y étaient pas soumises. Cet état de fait pouvait paraître paradoxal dans un pays comme la France qui a souvent fait figure de pionnière parmi les pays européens en matière de protection des personnes $[7,8]$. Cependant, une lecture attentive montre que ces études auraient dû en toute logique être soumises au CCTIRS et à la CNIL, ce qui n'avait pas été le cas, et qu'elles n'étaient donc pas en conformité avec la loi française. Rappelons que les études ayant pour objet de comparer des stratégies de soins utilisés en pratique courante (stratégies dites «soins courants ») doivent être soumises à un (PP mais ne sont pas considérées comme des recherches biomédicales au sens de la loi «Huriet-Sérusclat » et ne bénéficient pas de la procédure simplifiée.

Nous voudrions souligner qu'il serait judicieux d'utiliser le passage successif des études devant ces deux instances, le CCTIRS et la CNIL, 
comme une garantie de la qualité méthodologique d'une part, et de la protection des personnes contre la divulgation abusive de leurs données de santé d'autre part. Cette particularité de la loi française s'accorde parfaitement avec la déclaration d'Helsinki [9], et la combinaison CCTIRS-CNIL assure un niveau de protection scientifique et éthique largement équivalent au modèle «Institutional review board/Independant ethics committee » en vigueur aux États-Unis. À ce sujet, le CCTIRS est conscient de sa responsabilité pour éviter à des personnes de participer à des recherches qui n'auraient aucune chance d'aboutir et qui risqueraient de porter atteinte inutilement à leur vie privée. Ce point éthique est le motif principal des avis défavorables qui sont prononcés.

Enfin, nous souhaitons aborder un problème d'éthique auquel le comité a été plusieurs fois confronté. II arrive de manière non exceptionnelle qu'un projet de recherche relève de notre point de vue de la loi Huriet-Sérusclat mais que les responsables nous demandent notre avis comme s'il s'agissait d'une recherche observationnelle. Dans un tel cas, le comité ne peut exiger la soumission à un CPP, son avis étant purement consultatif. II use néanmoins de toutes les ressources dont il dispose pour persuader les demandeurs de se soumettre à la loi, et peut même aller jusqu'à émettre un avis défavorable en invoquant cette raison. Lorsque les demandeurs ne font simplement pas mention de cette soumission, le comité ne peut que donner un avis sur les critères qu'il est chargé d'apprécier et conseiller le passage devant un CPP. In fine c'est la CNIL qui statuera.

\section{Décret relatif à la conservation et à la préparation à des fins scientifiques d'éléments du corps humain}

Ce décret est paru le 10 août 2007. Le comité, qui avait été consulté sur cet avis de décret pris en application de la loi du 6 août 2004 relative à la bioéthique [10], n'a pu que constater que ses réserves sur la nécessité de recourir à sa compétence n’ont pas été retenues. En effet, le décret stipule que son avis doit être pris avant une décision d'opposition, de suspension ou d'interdiction d'activités déclarées de conservation et de préparation des éléments du corps humain, ainsi que pour les demandes d'autorisation des organismes qui réalisent ces activités en vue de leur cession pour un usage scientifique.

Les réserves du comité sont fondées sur la définition même de son rôle par la loi, qui est d'examiner des projets de recherche scientifique dans le domaine de la santé. La constitution d'une base de données ne peut être qualifiée de recherche et, lorsqu'un tel dossier lui est soumis, le comité ne donne pas d'avis formel mais rappelle qu'il examinera tout projet de recherche ultérieur utilisant les données de cette base. II ne peut donc logiquement assurer le contrôle des risques potentiels de rupture de la confidentialité générés par ce type de collection. Néanmoins, afin de se conformer au décret, le comité a résolu, après consultation des juristes du Ministère de l'enseignement et de la recherche, qu'il examinerait les dossiers que le Ministère lui soumettrait, et que, s'il constatait que les formalités auprès de la CNIL avaient été remplies, il ne rendrait aucun avis, celui-ci étant alors préjugé favorable.

\section{Accès aux données du SNIIRAM}

Dans le cadre de la création de l'Institut des données de santé (IDS) chargé de contrôler l'accès au système national d'information inter-régimes de l'assurance maladie (SNIIRAM), il est apparu que le recours aux données de l'assurance maladie pouvait être envisagé dans des projets de recherche soumis au comité consultatif. Des entretiens et des rencontres ont eu lieu afin d'harmoniser le rôle des différents organismes concernés.

Dans les cas de demandes d'accès au SNIIRAM où les projets relèvent du chapitre $X$ (traitement des données de santé à caractère personnel à des fins d'évaluation ou d'analyse des pratiques ou des activités de soin et de prévention), il a été décidé que I'IDS serait sollicité et que le CNIS serait informé, en plus de la déclaration obligatoire auprès de la CNIL.

Pour les projets de recherche dans le domaine de la santé, c'est la procédure classique impliquant le CCTIRS puis la CNIL qui doit être appliquée. En pratique, dans le cas où un projet de recherche signalerait qu'il nécessite l'accès aux données du SNIIRAM, le comité se prononce spécifiquement sur la pertinence d'un tel accès au regard de la finalité du projet afin que I'IDS et la CNIL en soient informés.

\section{Les études ayant pour objectif la promotion d'un produit de santé}

C'est le cas en particulier des études portant sur des médicaments ayant obtenu l'autorisation de mise sur le marché (études post-AMM). Certaines sont demandées par une autorité de santé, et le Comité les examine alors avec un œil particulièrement favorable sachant que la méthodologie sera de toute façon évaluée par cette autorité, mais vérifie que l'étude a été effectivement demandée et que le protocole ne dépasse pas largement les exigences de la dite autorité.

Pour les autres études dont l'objectif principal n'est manifestement pas d'augmenter les connaissances mais de faire la promotion d'un produit de santé, le Comité répond favorablement si l'étude s'accompagne d'un authentique projet de recherche satisfaisant aux exigences méthodologiques. En revanche, lorsque l'objectif apparaît purement commercial, le comité donne un avis défavorable lorsque la méthodologie proposée n'a aucune chance d'aboutir à l'objectif invoqué. En l'absence d'erreur méthodologique avérée, il ne donne pas d'avis formel mais recommande que l'étude soit réalisée sans aucune donnée identifiante, même si elle implique un suivi des personnes. En effet, ces études 
ne devraient pas être autorisées puisque le traitement de données de santé à caractère personnel est interdit par la loi en dehors de la recherche. Néanmoins, la CNIL peut autoriser le traitement si la sécurité juridique des transmissions de données est garantie, en considérant l'étude comme une évaluation de pratique de soin (chapitre $X$ ), à la condition d'un appauvrissement des données d'identification et d'une durée de conservation très brève des données à caractère personnel. Le comité a réalisé que son absence d'avis pouvait dans certains cas permettre la réalisation de «pseudo-recherches » en contradiction avec les principes éthiques qui le guident, et peut être alors amené à donner d'emblée un avis défavorable. Ces études s'apparentent bien souvent à des achats de prescription, les médecins recevant en général une compensation financière. Le Comité n'ayant pas pour mission de juger de la pertinence de la recherche, il ne peut donner un avis défavorable sur le seul motif qu'il suspecte un achat de prescription habillé en recherche. Par ailleurs, la limite entre une étude essentiellement destinée au marketing et une étude s'accompagnant d'un authentique projet de recherche n'est pas toujours facile à cerner. Ajoutons que les membres du comité sont toujours irrités par le temps perdu par l'examen de ces études dont l'intérêt économique est assez clair, mais l'intérêt scientifique extrêmement limité. On comprend alors pourquoi il existe parfois des divergences d'avis dans ce domaine. L'autorité compétente pour s'assurer que la rétribution des médecins est destinée à les indemniser de leur travail, et non de rétribuer la prescription d'un produit de santé, est le Conseil national de l'ordre des médecins avec lequel le comité est en contact régulier dans l'objectif d'une meilleure concertation pour assainir les pratiques dans ce domaine.

\section{Perspectives}

À l'occasion de son renouvellement, le comité a souhaité faire le point sur l'intérêt de son travail et la légitimité de ses avis. II est surtout inquiet de la grande méconnaissance de nombreux médecins et chercheurs sur la législation en matière de traitement des données à caractère personnel. La rédaction du présent article participe à cet effort de communication qui nous semble indispensable.
Il nous paraît cependant important de souligner que si l'information atteignait réellement son but, il est probable que le comité serait dans l'impossibilité de faire face à l'afflux de dossiers, compte tenu du faible nombre d'experts et du peu de moyens mis à sa disposition par le Ministère de l'enseignement supérieur et de la recherche. Le mode d'organisation actuel devrait alors être complètement revu, mais cette difficulté ne doit en aucun cas être un frein à la diffusion des règles sur le traitement de données à caractère personnel en matière de recherche dans le domaine de la santé. $\diamond$

Treatment of information on personal health data

for research purposes: nobody should ignore the law

\section{REMERCIEMENTS}

Les membres du comité tiennent à remercier Mesdames Michèle Guillemot, Jany Delhay et Claudine Poudroux pour leur assistance précieuse dans la conduite et l'organisation des expertises. Ils sont très reconnaissants à Madame Jeanne Bossi pour sa relecture attentive du manuscrit et ses remarques constructives.

\section{RÉFÉRENCES}

1. Loi n $78-17$ du 6 janvier 1978 , relative à l'informatique, aux fichiers et aux libertés.

2. Loi $n^{\circ} 94-548$ du $1^{\text {er }}$ juillet 1994 , relative aux traitements de données nominatives ayant pour fin la recherche dans le domaine de la santé et modifiant la loi n ${ }^{\circ} 78-17$ du 6 janvier 1978 , relative à l'informatique, aux fichiers et aux libertés.

3. Loi n² 2004-801 du 6 août 2004, relative à la protection des personnes physiques à l'égard des traitements de données à caractère personnel et modifiant la loi $n^{\circ} 78-17$ du 6 janvier 1978 , relative à l'informatique, aux fichiers et aux libertés.

4. Loi n $88-1138$ du 20 décembre 1988, relative à la protection des personnes qui se prêtent à des recherches biomédicales.

5. Bara C, Berr C, Blacher J, et al. Projet de loi relatif à la protection des personnes physiques à l'égard des traitements de données à caractère personnel. Position du Comité consultatif sur le traitement de l'information en matière de recherche dans le domaine de la santé (CCTIR). Rev Epidemiol Sante Publ $2003 ; 51: 364-7$.

6. Lemaire F. Do all types of human research need ethics committee approval? Am J Respir Crit Care Med $2006 ; 174: 363-4$.

7. Chneiweiss H. De l'innovation en matière de médicaments à la thérapeutique : nouvelle donne éthique. Med Sci (Paris) $2004 ; 20: 259-60$.

8. Jaillon P, Demarez JP. L'histoire de la genèse de la loi Huriet-Sérusclat de décembre 1988 : Ioi sur la protection des personnes qui se prêtent à des recherches biomédicales. Med Sci (Paris) $2008 ; 24: 323-7$.

9. World medical association (WMA). Declaration of Helsinki. Ethical principles for medical research involving human subjects (online). 2004 october 9 (cited 2007 July 10). Available from : http://www.wma.net/e/policy/b3.htm

10. Loi 2004-800 du 6 août 2004 relative à la bioéthique.

11. Lemaire F. La mission des Comités de protection des personnes en France : ni éthique, ni scientifique? Med Sci (Paris) $2005 ; 21: 876-9$.
TIRÉS À PART

C. Bonaïti-Pellié 

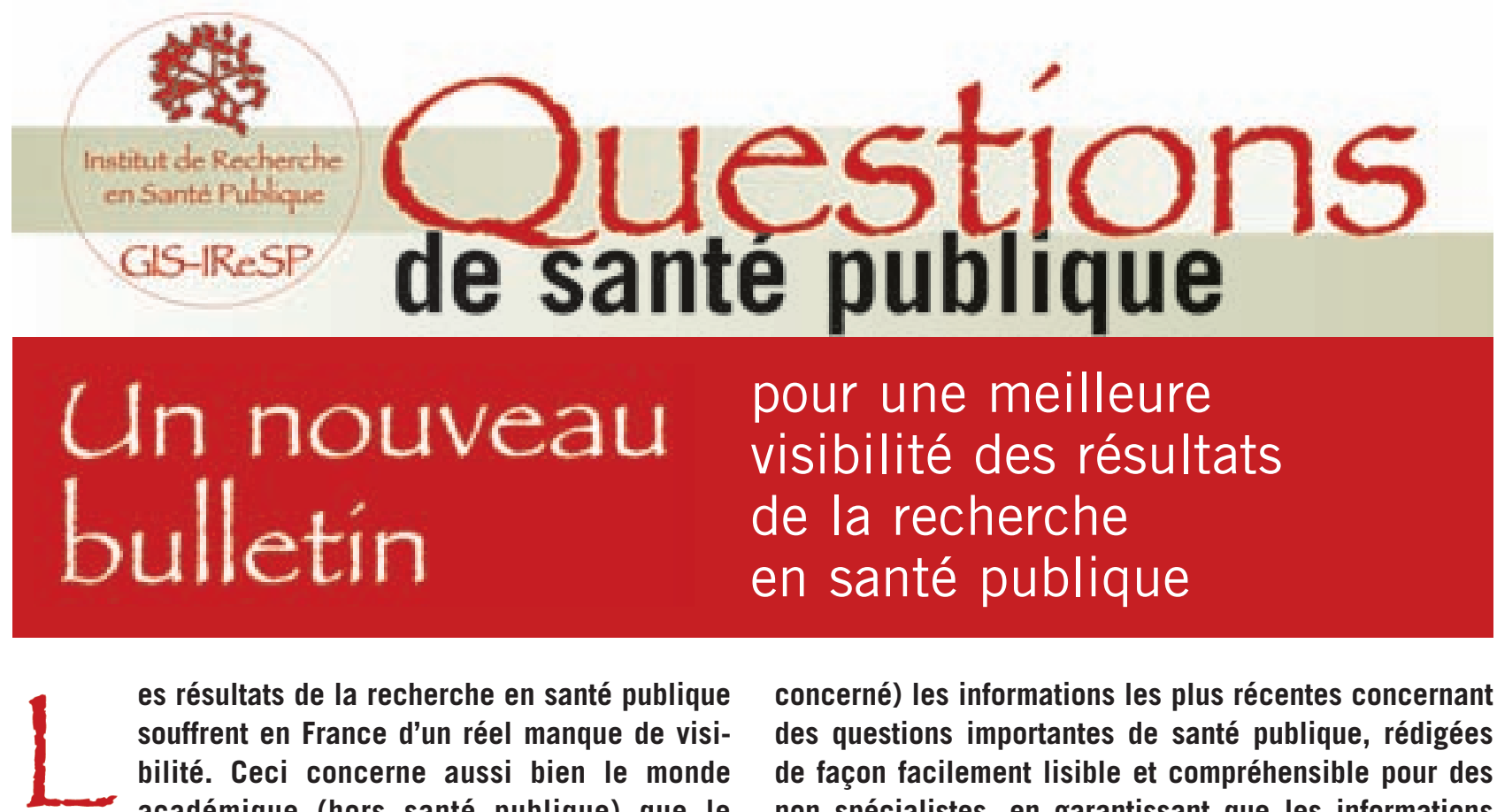

es résultats de la recherche en santé publique souffrent en France d'un réel manque de visibilité. Ceci concerne aussi bien le monde académique (hors santé publique) que le grand public et les décideurs. Pour pallier ce déficit, I'IReSP crée un bulletin à large diffusion intitulé "Questions de santé publique ", largement inspiré du bulletin mensuel d'information de l'INED « Populations et sociétés ". L'objectif éditorial est de porter à la connaissance d'un large public (enseignants, étudiants, journalistes, décideurs, milieux de la recherche, associations, public

concerné) les informations les plus récentes concernant des questions importantes de santé publique, rédigées de façon facilement lisible et compréhensible pour des non spécialistes, en garantissant que les informations publiées sont validées scientifiquement. La publications concernera des faits et non des positions. Au-delà de la présentation de résultats, cette publication devrait également avoir des qualités pédagogiques, permettant au lecteur de mieux comprendre comment sont formulées et abordées les questions de santé publique et quelles sont les limites de ces études.

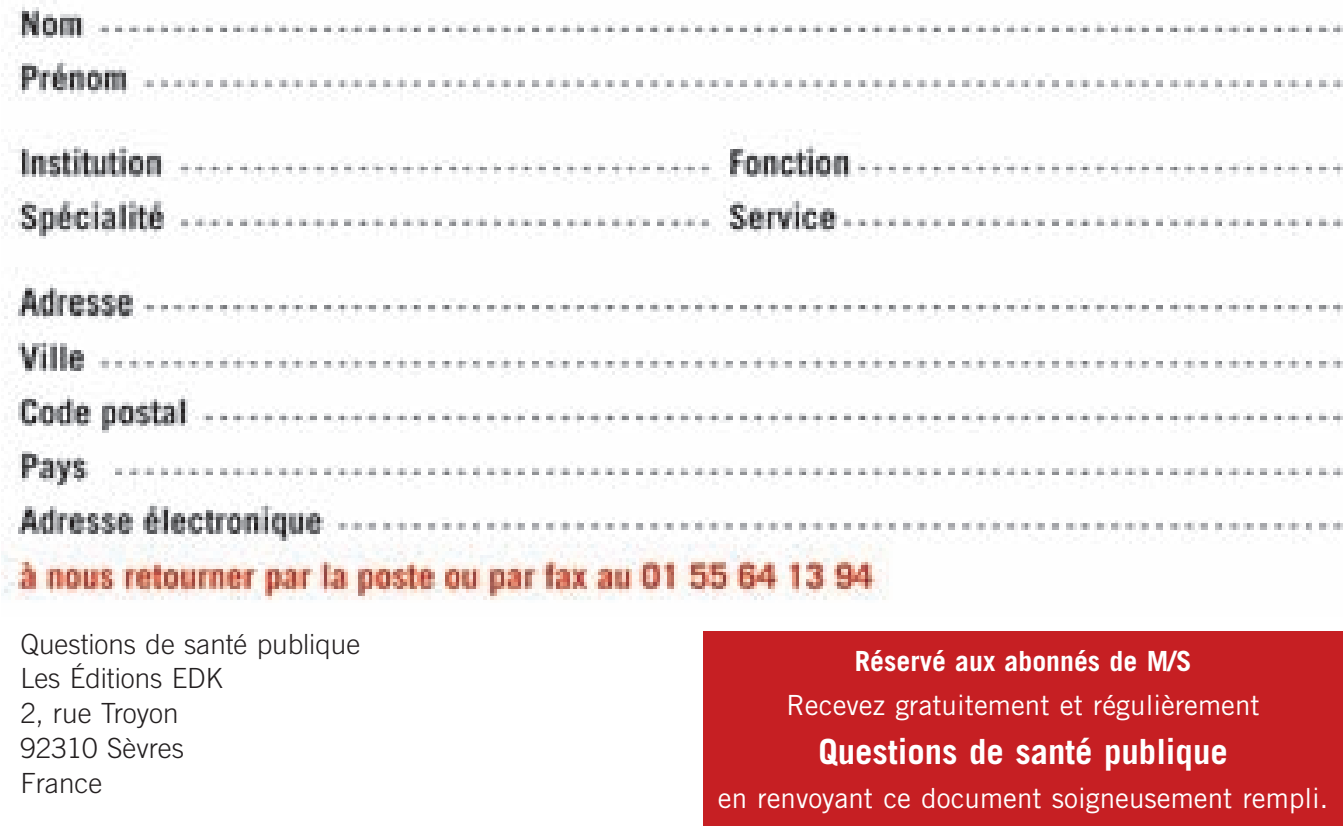

Questions de santé publique est une publication de l'Institut de Recherche en Santé Publique. I Directeur de la publication : Alfred Spira. I Rédacteur en chef : Nathalie de Parseval. I Une réalisation des Éditions EDK. 